\title{
Left anterior temporal cortex actively engages in speech perception: A direct cortical stimulation study.
}

\section{$\operatorname{AUTHOR}(\mathrm{S}):$}

Matsumoto, Riki; Imamura, Hisaji; Inouchi, Morito; Nakagawa, Tomokazu; Yokoyama, Yohei; Matsuhashi, Masao; Mikuni, Nobuhiro; ... Fukuyama, Hidenao; Takahashi, Ryosuke; Ikeda, Akio

\section{CITATION:}

Matsumoto, Riki ...[et al]. Left anterior temporal cortex actively engages in speech perception: A direct cortical stimulation study.. Neuropsychologia 2011, 49(5): 1350-1354

\section{ISSUE DATE:}

2011-04

URL:

http://hdl.handle.net/2433/141342

\section{RIGHT:}

(c) 2011 Elsevier Ltd.; この論文は出版社版でありません。引用の際には 出版社版をご確認ご利用ください。; This is not the published version. Please cite only the published version. 
Title

Left anterior temporal cortex actively engages in speech perception: a direct

cortical stimulation study

Riki Matsumoto ${ }^{1}$, Hisaji Imamura ${ }^{1}$, Morito Inouchi ${ }^{1}$, Tomokazu Nakagawa ${ }^{1}$,

Yohei Yokoyama ${ }^{2}$, Masao Matsuhashi ${ }^{3}$, Nobuhiro Mikuni ${ }^{2}$, Susumu Miyamoto ${ }^{2}$,

Hidenao Fukuyama ${ }^{3}$, Ryosuke Takahashi $^{1}$, Akio Ikeda $^{1}$

1 Department of Neurology, 2 Department of Neurosurgery, 3 Human Brain

Research Center, Kyoto University Graduate School of Medicine, Kyoto, Japan

Address correspondence to:

Riki Matsumoto, M.D., Ph.D.

Department of Neurology, Kyoto University Graduate School of Medicine

54 Kawahara-cho, Shogoin, Sakyo-ku, Kyoto, 606-8507, Japan

Tel: $+81-75-751-3772$ 
Matsumoto 2

Fax: $+81-75-751-9416$

Email: matsumot@kuhp.kyoto-u.ac.jp

Shortened title: Left anterior temporal cortex for speech perception

Total word count:

Number of figures and tables: $\quad 2$ figures and 2 tables 


\section{Abstract}

Recent neuroimaging studies proposed the importance of the anterior auditory pathway for speech comprehension. Its clinical significance is implicated by semantic dementia or pure word deafness. Neurodegenerative or cerebrovascular nature, however, precluded precise localization of the cortex responsible for speech perception. Electrical cortical stimulation could delineate such localization by producing transient, functional impairment. We investigated engagement of the left anterior temporal cortex in speech perception by means of direct electrical cortical stimulation. Subjects were two partial epilepsy patients, who underwent direct cortical stimulation as a part of invasive presurgical evaluations. Stimulus sites were coregistered to presurgical 3D-MRI, and then to MNI standard space for anatomical localization. Separate from the posterior temporal language area, electrical cortical stimulation revealed a well-restricted language area in the anterior part of the superior temporal sulcus and gyrus (aSTS/STG) in both patients. Auditory sentence comprehension was impaired upon electrical stimulation of aSTS/STG. In one patient, additional investigation 
Matsumoto 4

revealed that the functional impairment was restricted to auditory sentence comprehension with preserved visual sentence comprehension and perception of music and environmental sounds. Both patients reported that they could hear the voice but not understand the sentence well (e.g., heard as a series of meaningless utterance). The standard coordinates of this restricted area at left aSTS/STG well corresponded with the coordinates of speech perception reported in neuroimaging activation studies in healthy subjects. The present combined anatomo-functional case study, for the first time, demonstrated that aSTS/STG in the language dominant hemisphere actively engages in speech perception.

\section{Key words}

speech perception; language; electrical cortical stimulation; superior temporal sulcus; epilepsy surgery 


\section{Introduction}

Most stroke studies of aphasia localized verbal comprehension to posterior temporal and inferior parietal cortices, while evidence from focal cortical neurodegenerative disorder, semantic dementia instead implicates anterior temporal cortex (Gorno-Tempini et al., 2004). Recent functional neuroimaging studies in normal subjects support the presence of parallel, anterior and posterior auditory streams from the primary auditory cortex for speech comprehension (Scott \& Johnsrude, 2003). The importance of anterior temporal cortex in verbal comprehension is further supported by pure word deafness, a rare form of auditory agnosia characterized by impairment of speech perception with preserved perception of music and environmental sounds (Buchman et al., 1986). The majority of cases have resulted from lesions in the bilateral or left superior temporal gyrus, but the cerebrovascular insult precluded precise localization of the cortex responsible for speech perception. Direct electrical stimulation of the cortex has been a gold standard measure to map eloquent cortical areas with a relatively good spatial resolution $(\sim 1 \mathrm{~cm})$ in patients who are 
candidate for surgical treatment of intractable partial epilepsy (Lüders et al., 1987). In contrast to neuroimaging activation studies, electrical cortical stimulation can delineate the cortex responsible for a particular task by making functional impairment. By means of direct electrical cortical stimulation, we demonstrated that a restricted region of the anterior temporal cortex actively engaged in speech perception in two epilepsy patients.

\section{Material and methods}

\subsection{Subjects}

Two patients were enrolled in this study. Subject 1 was a 30 -year-old right-handed woman with intractable left mesial temporal lobe epilepsy. The patient had a prolonged febrile seizure at 6 months old. Her habitual seizures started at age of 7 years and consisted of abdominal aura and complex partial seizures with hand and oral automatisms. Brain MRI showed atrophy and increased signal intensity (FLAIR) in the left hippocampus. Prolonged video-EEG monitoring showed interictal and ictal discharges in the left anterior 
temporal region. Neuropsychology batteries showed lower border of normal intelligence and normal language function (WAIS-III total IQ 88, verbal IQ 84, performance IQ 95; WAB aphasia quotient 96). Wada test revealed language dominancy in the left hemisphere. The patient underwent invasive evaluations with subdural electrodes in order to localize the epileptic focus and perform functional cortical mapping. The subdural electrodes were placed in the left lateral and basal temporal areas. The patient finally underwent tailored resection of the medial temporal structures and the pathology was hippocampal sclerosis. The patient has been seizure free for two years except for very rare abdominal auras.

Subject 2 was a 32-year-old woman with intractable partial epilepsy. No remarkable perinatal or past history was noted. Her habitual seizures started at age of 15 years and consisted of complex partial seizures that were infrequently preceded by auditory or visual auras. Brain MRI was unremarkable. Video-EEG monitoring and magnetoencephalography revealed interictal discharges in the left lateral temporal and parietal regions and ictal EEG onset in the left lateral 
Matsumoto 8

temporal region. Neuropsychology batteries showed mildly impaired intelligence and normal language function (WAIS-R total IQ 70, verbal IQ 68, performance IQ 78; WAB aphasia quotient 98.3). Wada test revealed language dominancy in the left hemisphere. The patient underwent invasive evaluations with subdural electrodes in order to localize the epileptic focus and perform functional cortical mapping. The subdural electrodes were placed in the left lateral temporo-parietal and basal temporal areas. Ictal onset zone was identified in the basal temporal region and the posterior part of the lateral temporo-parietal region. The patient finally underwent right standard anterior temporal lobectomy and corticectomy of the posterior part of the lateral temporo-parietal area. The pathology was focal cortical dysplasia (Palmini's classification type IA). The patient has been seizure free for one year.

This study was approved by the ethics committee of our institute (No. 79) and the patients gave written informed consent.

\subsection{Electrical Cortical Stimulation}


Matsumoto 9

Electrical cortical stimulation was performed with subdural electrodes

(platinum-made, inter-electrode distance of $1 \mathrm{~cm}$, recording diameter of $2.3 \mathrm{~mm}$,

ADTECH, WI). Repetitive square-wave electric currents of alternating polarity

with a pulse width of $0.3 \mathrm{~ms}$ and a frequency of $50 \mathrm{~Hz}$ were delivered through a

pair of electrodes for 4-5 s (Electrical stimulator S-7203, Nihon Kohden, Tokyo,

Japan). Details of the methodology for cortical stimulation and the subsequent

cortical mapping have been described elsewhere (Lüders et al., 1987;

Matsumoto et al., 2004; Usui et al., 2005). After confirming the absence of

positive (e.g., tonic contraction) and negative (e.g., impairment of rapid

alternating movements) tongue motor responses, a series of language battery

was tested during 4-5 sec trials with electrical stimulation at the intensity of

11-15 mA. The language battery in their native language of Japanese consisted

of paragraph reading, picture naming, kana word reading, kanji word reading,

and auditory sentence comprehension. In the paragraph reading, subjects read

aloud a part of children's story consisting of 100-150 words (kana mixed with

kanji). For the picture naming, non-animate objects (e.g., watch), each of which 
was printed in black on a white card, were shown to the subjects. Test items were carefully chosen from materials that are commonly used in daily life of ordinary Japanese so that the performance of subjects would not be affected by the familiarity with the items. In reading kanji and kana words, the same items used in picture naming were employed. Auditory sentence comprehension task consisted of making gestures following simple one-sentence commands (e.g., open your mouth, presented by the examiner's voice). In patient 1 , additional tasks were examined in the anterior temporal cortex to further clarify the language function: visual sentence comprehension (i.e., visual presentation of the same sentence used in the auditory sentence comprehension task), perception of the classical music and non-linguistic sounds (e.g., bell, waterfall). These tasks were not performed in patient 2 due to frequent afterdischarges.

The majority of electrical stimulation was performed in a bipolar fashion (i.e., stimulation at the adjacent two electrodes). In patient 2, monopolar stimulation in reference to an electrode in the nonfunctional cortex was also performed in the posterior temporal region to further localize the language areas 
for the clinical purpose. We judged the impaired behaviors as significant when the findings were reproducible in the absence of afterdischarges. In case of very brief afterdischarges such as several seconds elicited, if they were so brief and behaviors were impaired, it strongly suggested that the cortices at or very close to the stimulus site were responsible for the particular cortical function.

\subsection{Anatomical localization of subdural electrodes in individual and}

\section{standard space}

A magnetization-prepared rapid gradient echo (MPRAGE) sequence was applied for anatomical T1-weighted volume data acquisition. MPRAGE volumetric scan was performed before and after implantation of subdural electrodes as a part of presurgical evaluations. In the volumetric scan taken after implantation, the location of each electrode was identified on the $2 \mathrm{D}$ slices using its signal void due to the property of platinum alloy (Matsumoto et al., 2004). Electrodes were non-linearly co-registered to the patient MRI (MPRAGE) taken before implantation, and then to MNI standard space (ICBM-152) using FNIRT 
(www.fmrib.ox.ac.uk/fsl/fnirt/). Talairach coordinates of electrodes were

converted from MNI coordinates by icbm2tal transform

(http://brainmap.org/icbm2tal/) (Lancaster et al., 2007).

\section{Results}

\subsection{Functional cortical mapping}

In patient 1 , stimulation of the left lateral temporal area revealed two separate

areas with language impairment: one at the anterior part of the superior temporal

sulcus and gyrus (aSTS/STG) and the other at the posterior part of the middle

temporal gyrus (pMTG) (Fig. 1). While stimulation of pMTG elicited global

aphasia (i.e., impairment in all tasks), that of aSTS/STG only elicited impaired

auditory sentence comprehension. The patient reported that speech was heard

as a series of meaningless utterance, though she could recognize that the

utterance belonged to the examiner's voice. Additional tasks were examined

during stimulation to further clarify the language function at aSTS/STG. Upon

visual presentation of the same sentence, the patient could read and understand 
the sentence, and performed the written order correctly. Perception of classical music and non-linguistic sounds was not impaired.

In patient 2, stimulation of the left lateral temporal area also revealed two separate areas with language impairment: one at aSTS/STG and the other at the posterior part of the superior (pSTG), middle (pMTG1-3) and inferior temporal (pITG) gyri (Fig. 1). Stimulation of aSTS/STG showed selective impairment of auditory sentence comprehension, while paragraph reading and picture naming was spared. Due to very frequent afterdischarges elicited, the reproducibility of functional impairment at aSTS/STG could be confirmed at least partially in the second trial with a short run of afterdischarges in the adjacent electrodes. On the other hand, stimulation of the posterior language areas elicited global aphasia (pMTG1, pMTG3), impairment of auditory sentence comprehension (pSTG, pMTG2) or selective impairment of visual comprehension (pITG). As for the patient's introspection during aSTS/STG stimulation, the patient reported that she could hear the voice but failed to understand the contents to perform the gesture. 
The results of electrical cortical stimulation were summarized in Table

1.

\subsection{Standard coordinates of anterior temporal cortex associated with}

impairment of speech comprehension

Coordinates of the aSTS/STG electrodes were shown in MNI standard space

(Fig. 2). aSTS/STG electrodes were located in close proximity between the two patients (4-7 mm difference, especially $2 \mathrm{~mm}$ difference in the anterior-posterior axis). The coordinates for speech perception in neuroimaging studies in healthy subjects were shown in the same figure for comparison (Friederici et al., 2003;

Liebenthal et al., 2005; Narain et al., 2003; Scott et al., 2000; Spitsyna et al., 2006). The coordinates of aSTS/STG electrodes well corresponded with the coordinates for speech perception in the anterior temporal cortex. These coordinates were summarized in Table 2 together with their specific functions.

\section{Discussion}


Matsumoto 15

Recent neuroimaging studies revealed hierarchical organization of the anterior

auditory stream for speech comprehension; presumably speech is initially

analyzed for its physical features in the middle part of the bilateral superior

temporal gyrus (STG), then processed in the left anterior part of STS/STG (left

aSTS/STG) for speech intelligibility, and finally in the more anterior and ventral

temporal areas for lexical-semantic retrieval (Liebenthal et al., 2005; Scott et al.,

2000; Scott \& Johnsrude, 2003). In contrast to neuroimaging activation studies,

electrical cortical stimulation can delineate the cortex responsible for a particular

task by making functional impairment. The functional interference is temporary

( $\sim \mathrm{sec})$, discretely focal $\left(\sim 1 \mathrm{~cm}^{2}\right)$, and in sharp contrast to chronic stroke

lesions that are relatively large and usually associated with cortical plastic

compensation (Nathan et al., 1993). In the present study, electrical cortical

stimulation of the left aSTS/STG produced impairment of auditory sentence

comprehension in both patients. In patient 1, functional impairment was

restricted to auditory sentence comprehension with preserved visual sentence

comprehension and perception of music and environmental sounds. Although 
frequent afterdischarges resulted in partial confirmation of reproducibility of the responses and prevented us from additional examination performed in patient 1 , we consider that patient 2 successfully reproduced the role of aSTS/STG in speech perception because i) both patients showed similar introspection during stimulation, ii) standard coordinates of the aSTS/STG electrodes were very concordant between the two patients (difference $<7 \mathrm{~mm}$, especially $2 \mathrm{~mm}$ difference in the anterior-posterior axis), and iii) the functional localization was restricted to a single pair of electrodes in both patients (no language impairment upon stimulation at the adjacent pairs of electrodes).

Regarding the specific role in speech comprehension, left aSTS/STG may engage in syllable discrimination. Although auditory syllable discrimination was not investigated in the present patients, this hypothesis is less likely since the aSTS/STG coordinate was approximately $2.5 \mathrm{~cm}$ anterior to that of syllable discrimination deficit with electrical stimulation in epilepsy patients having normal listening ability (average coordinate: $x, y, z=-65,-29,9)$ (Boatman \& Miglioretti, 2005). Absence of electrocorticographic gamma increase in left 
aSTS/STG during an auditory sublexical speech (e.g., syllable) discrimination paradigm (Sinai et al., 2009) further supports the role of left aSTS/STG in higher-level linguistic processing. The standard coordinates of aSTS/STG electrodes were in good accordance with the coordinates for speech perception in neuroimaging activation studies (Fig. 2, Table 2) (Friederici et al., 2003; Liebenthal et al., 2005; Narain et al., 2003; Scott et al., 2000; Spitsyna et al., 2006). These activation studies attempted to highlight the specific functions in speech perception by employing various manipulations (Table 2). The majority of studies ascribed the sentence-level speech comprehension to left aSTS/STG (Friederici et al., 2003; Narain et al., 2003; Scott et al., 2000; Spitsyna et al., 2006), while Leibenthal et al. (2005) found that left aSTS/STG is also involved in phonemic perception together with the middle part of left STG. Taken together with the patients' introspection that they could not understand the sentence well, it is most likely that the impairment of speech comprehension, upon stimulation of left aSTS/STG, resulted from speech perception itself (possibly at the sentence-level comprehension) or a disruption of access to the lexical-semantic 
system from speech perception in the present patients. Systematic attempts have not been made previously with electrical cortical stimulation, and the present study for the first time clarified that aSTS/STG in the language dominant hemisphere actively engages in speech perception. Though the epileptic focus was away from aSTS/STG by $4-5 \mathrm{~cm}$ in both patients, the observation was made in two patients and further case accumulation is warranted. Since speech perception consists of a very complex set of processes, more specific tasks, such as perception of isolated syllables/words or a task modulating the grammatical complexity of the sentence, would clarify the specific role of aSTS/STG in speech perception. Whether the resection of this restricted area results in permanent deficit or not is clinically important to be solved. Careful observation after selective resection of this area would elucidate the degree of cortical plastic compensation.

\section{Acknowledgements}


Matsumoto 19

This work was partly supported by Grants-in-Aid for Scientific Research (C)

20591022 from the Ministry of Education, Culture, Sports, Science and

Technology of Japan (MEXT), the Research Grants from the Japan Epilepsy

Research Foundation, and the Research Grant for the Treatment of Intractable

Epilepsy (22-1) from the Japanese Ministry of Health, Labour and Welfare.

Conflicts of Interests: None declared. 


\section{References}

Boatman D.F., Miglioretti D.L. (2005). Cortical sites critical for speech

discrimination in normal and impaired listeners. J Neurosci, 25(23),

$5475-80$.

Buchman A.S., Garron D.C., Trost-Cardamone J.E., Wichter M.D., Schwartz M.

(1986). Word deafness: one hundred years later. J Neurol Neurosurg

Psychiatry, 49(5), 489-99.

Friederici A.D., Ruschemeyer S.A., Hahne A., Fiebach C.J. (2003). The role of

left inferior frontal and superior temporal cortex in sentence

comprehension: localizing syntactic and semantic processes. Cereb

Cortex, 13(2), 170-7.

Gorno-Tempini M.L., Dronkers N.F., Rankin K.P., Ogar J.M., Phengrasamy L.,

Rosen H.J., Johnson J.K., Weiner M.W., Miller B.L. (2004). Cognition and anatomy in three variants of primary progressive aphasia. Ann Neurol, 55(3), 335-46.

Lancaster J.L., Tordesillas-Gutierrez D., Martinez M., Salinas F., Evans A., Zilles 
K., Mazziotta J.C., Fox P.T. (2007). Bias between MNI and Talairach coordinates analyzed using the ICBM-152 brain template. Hum Brain Mapp, 28(11), 1194-205.

Liebenthal E., Binder J.R., Spitzer S.M., Possing E.T., Medler D.A. (2005).

Neural substrates of phonemic perception. Cereb Cortex, 15(10), 1621-31.

Lüders H., Lesser R., Dinner D., Morris H., Hahn J., Friedman L., et al. (1987).

Commentary: chronic intracranial recording and stimulation with subdural electrodes. In Engel JJ (Ed.), Surgical treatment of the epilepsies (pp. 297-321). New York: Raven Press.

Matsumoto R., Nair D.R., LaPresto E., Najm I., Bingaman W., Shibasaki H., Lüders H.O. (2004). Functional connectivity in the human language system: a cortico-cortical evoked potential study. Brain, 127(Pt 10), 2316-30.

Narain C., Scott S.K., Wise R.J., Rosen S., Leff A., Iversen S.D., Matthews P.M. (2003). Defining a left-lateralized response specific to intelligible speech 
using fMRI. Cereb Cortex, 13(12), 1362-8.

Nathan S.S., Sinha S.R., Gordon B., Lesser R.P., Thakor N.V. (1993).

Determination of current density distributions generated by electrical stimulation of the human cerebral cortex. Electroencephalogr Clin Neurophysiol, 86(3), 183-92.

Scott S.K., Blank C.C., Rosen S., Wise R.J. (2000). Identification of a pathway for intelligible speech in the left temporal lobe. Brain, $123 \mathrm{Pt}$ 12, 2400-6.

Scott S.K., Johnsrude I.S. (2003). The neuroanatomical and functional organization of speech perception. Trends Neurosci, 26(2), 100-7.

Sinai A., Crone N.E., Wied H.M., Franaszczuk P.J., Miglioretti D., Boatman-Reich D. (2009). Intracranial mapping of auditory perception: event-related responses and electrocortical stimulation. Clin Neurophysiol, $120(1), 140-9$.

Spitsyna G., Warren J.E., Scott S.K., Turkheimer F.E., Wise R.J. (2006). Converging language streams in the human temporal lobe. J Neurosci, 26(28), 7328-36. 
Matsumoto 23

Usui K., Ikeda A., Takayama M., Matsuhashi M., Satow T., Begum T., et al.

(2005). Processing of Japanese morphogram and syllabogram in the left

basal temporal area: electrical cortical stimulation studies. Brain Res

Cogn Brain Res. 24(2), 274-83. 


\section{Figure legend}

Figure 1

Results of electrical cortical stimulation in the lateral temporal area. Anatomical location of each subdural electrode was displayed on 3D MRI taken before implantation of subdural electrodes by means of non-linear transformation. Green dots represent locations of subdural electrodes. Only the electrodes though which cortical stimulation was performed for language mapping in the lateral temporal area were shown. Electrodes showing language impairment upon stimulation were highlighted by red.

aSTS/STG = anterior part of the superior temporal sulcus and gyrus; $\mathrm{pSTG}=$ posterior part of the superior temporal gyrus; pMTG = posterior part of the middle temporal gyrus; pITG = posterior part of the inferior temporal gyrus .

Figure 2

Comparison of aSTS/STG areas responsible for speech perception in MNI standard space: Red (aSTS/STG electrodes in patient 1), pink (aSTS/STG 
Matsumoto 25

electrodes in patient 2), yellow (Liebenthal et al., 2005), blue (Scott et al., 2000), light blue (Friederici et al., 2003), green (Spitsyna et al., 2006), orange (Narain et al., 2003). For presentation purpose, electrode locations and peak foci of activation studies were collapsed onto a representative brain slice at $-54 \mathrm{~mm}$ from midline. 
Table1

Results of high frequency electrical cortical stimulation in the lateral temporal cortex

\begin{tabular}{|c|c|c|c|c|c|c|c|c|}
\hline $\begin{array}{l}\text { Stimulus site } \\
\text { (intensity, duration) }\end{array}$ & $\begin{array}{l}\text { auditory sentence } \\
\text { comprehension }\end{array}$ & $\begin{array}{c}\text { paragraph } \\
\text { reading }\end{array}$ & $\begin{array}{l}\text { picture } \\
\text { naming }\end{array}$ & $\begin{array}{c}\text { Kanji word } \\
\text { reading }\end{array}$ & $\begin{array}{l}\text { Kana word } \\
\text { reading }\end{array}$ & $\begin{array}{l}\text { visual sentence } \\
\text { comprehension }\end{array}$ & $\begin{array}{c}\text { music } \\
\text { perception }\end{array}$ & $\begin{array}{l}\text { non-linguistic } \\
\text { sound } \\
\text { perception }\end{array}$ \\
\hline \multicolumn{9}{|l|}{ Patient 1} \\
\hline aSTS/STG (15 mA, $5 \mathrm{sec})$ & $x$ & $\bigcirc$ & $\bigcirc$ & $\bigcirc$ & $\bigcirc$ & $\bigcirc$ & $\bigcirc$ & $\bigcirc$ \\
\hline pMTG (13 mA, $4 \mathrm{sec})$ & $x$ & $x$ & $x$ & $x$ & $x$ & - & - & - \\
\hline \multicolumn{9}{|l|}{ Patient 2} \\
\hline aSTS/STG (15 mA, $5 \mathrm{sec})$ & $\times$ & $\bigcirc$ & $\bigcirc$ & - & - & - & - & - \\
\hline pSTG (15 mA, $5 \mathrm{sec})$ & $x$ & $\bigcirc$ & $\bigcirc$ & - & - & - & - & - \\
\hline pMTG1 (13 mA, $5 \mathrm{sec})$ & $x$ & $x$ & $x$ & - & - & - & - & - \\
\hline pMTG2 (14 mA, $5 \mathrm{sec})$ & $x$ & $\bigcirc$ & $\bigcirc$ & - & - & $x$ & - & - \\
\hline pMTG3 (15 mA, $5 \mathrm{sec})$ & $x$ & $x$ & $x$ & - & - & - & - & - \\
\hline pITG (10 mA, $4 \mathrm{sec})$ & 0 & $x$ & $x$ & - & - & - & - & - \\
\hline
\end{tabular}

o = no impairment ; $x=$ impaired during stimulation ; - = not tested $;$ aSTS/STG $=$ anterior part of the supetrior temporal sulcus/gyrus

pSTG $=$ posterior part of the superior temporal gyrus $;$ pMTG = posterior part of the middle temporal gyrus $;$ pITG = posterior part of the inferior temporal gyrus 
Table 2

Standard coordinates for speech perception in the anterior temporal cortex

\begin{tabular}{|c|c|c|c|}
\hline Study & $\begin{array}{c}\text { Function } \\
\text { (patient's introspection during stimulation } \\
\text { or contrasts used in imaging studies) }\end{array}$ & $\begin{array}{l}\text { MNI coordinate } \\
\quad(x \text { y z })\end{array}$ & $\begin{array}{l}\text { Talairach coordinate } \\
\qquad(x \text { y } z)\end{array}$ \\
\hline \multicolumn{4}{|c|}{ Electrical Cortical Stimulation } \\
\hline $\begin{array}{l}\text { aSTS/STG electrodes } \\
\text { (patient 1) }\end{array}$ & $\begin{array}{l}\text { speech perception } \\
\text { ("Speech was heard as a series of meaningless utterence") }\end{array}$ & $\begin{array}{lll}-62 & 4 & -10 \\
-66 & -6 & -6\end{array}$ & $\begin{array}{lll}-60 & 4 & -6 \\
-64 & -6 & -3\end{array}$ \\
\hline $\begin{array}{l}\text { aSTS/STG electrodes } \\
\text { (patient 2) }\end{array}$ & $\begin{array}{l}\text { speech perception } \\
\text { ("I could hear the voice but failed to understand the contents } \\
\text { of the sentence") }\end{array}$ & $\begin{array}{lll}-62 & 6 & -6 \\
-66 & -4 & 0\end{array}$ & $\begin{array}{lll}-60 & 6 & -2 \\
-64 & -4 & 3\end{array}$ \\
\hline \multicolumn{4}{|l|}{ Neuroimaging studies } \\
\hline Friederici et al., 2003 & $\begin{array}{l}\text { Syntactic processing in sentence comprehension } \\
\text { (syntactically violated sentences vs. correct sentences) }\end{array}$ & $\begin{array}{lll}-55 & -1 & -3\end{array}$ & $\begin{array}{lll}-53 & -1 & 0\end{array}$ \\
\hline Liebenthal et al., 2005 & $\begin{array}{c}\text { phonemic perception } \\
\text { (speech syllables vs. non-phonemic sounds) }\end{array}$ & $\begin{array}{lll}-62 & -8 & -6\end{array}$ & $\begin{array}{lll}-60 & -8 & -3\end{array}$ \\
\hline Narain et al., 2003 & $\begin{array}{l}\text { sentence-level speech comprehension } \\
\text { (short sentences vs. controlled acoustic signals) }\end{array}$ & $-56-6-20$ & $-53-5-15$ \\
\hline Scott et al., 2000 & $\begin{array}{l}\text { sentence-level speech comprehension } \\
\text { (short sentences vs. controlled acoustic signals) }\end{array}$ & $\begin{array}{lll}-54 & 6 & -16 \\
-66 & -12 & -12\end{array}$ & 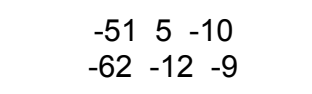 \\
\hline Spitsyna et al., 2006 & $\begin{array}{l}\text { setence-level speech comprehension } \\
\text { (spoken narratives vs. controlled acoustic signals) }\end{array}$ & $-58-6-16$ & $-55-6-12$ \\
\hline
\end{tabular}

Activation peaks were plotted in the standard coordinates for neuroimaging studies.

Icbm2tal transform was used for transformation between MNI and Talairach coordinates (Lancaster et al., 2007). 


\section{Functional cortical mapping of lateral temporal language areas}
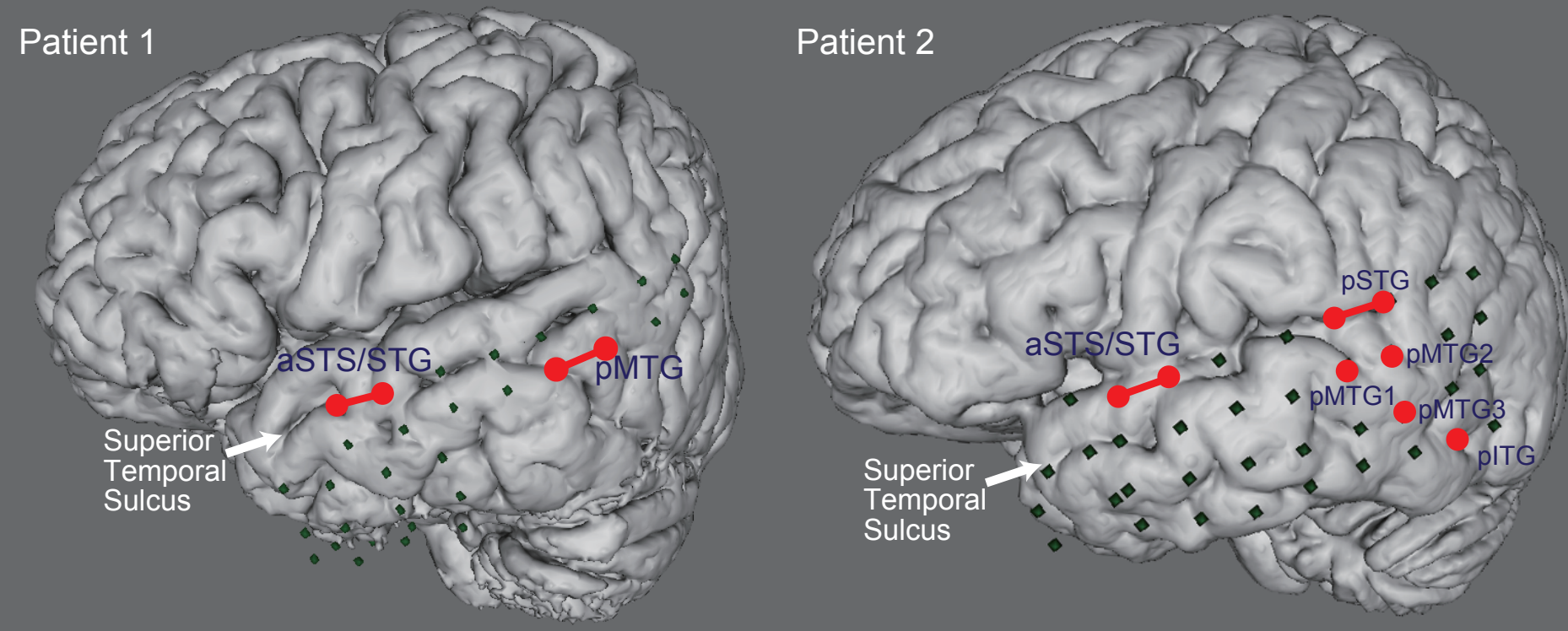


\section{MNI coordinates for speech perception}

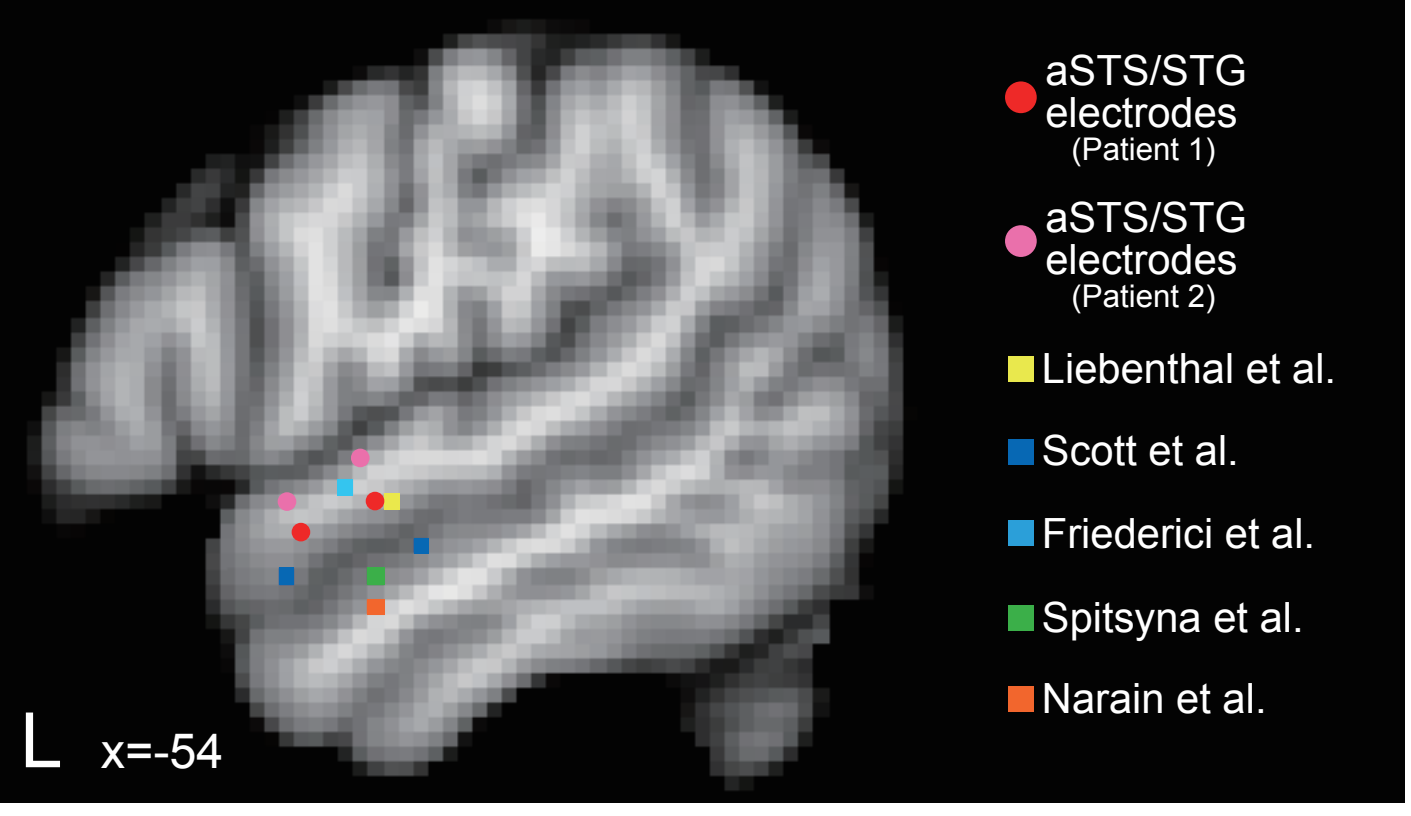

